\title{
p-ADIC CONTINUED FRACTIONS OF LIOUVILLE TYPE
}

\author{
VICHIAN LAOHAKOSOL AND PATCHARA UBOLSRI
}

(Communicated by Larry J. Goldstein)

\begin{abstract}
A p-adic analogue of a recent algebraic independence criterion of Adams is proved. It is then applied to construct Liouville type $p$-adic continued fractions of the kinds first considered by Ruban, and by Schneider.
\end{abstract}

1. Introduction. Various attempts to create a satisfactory continued fraction theory in the $p$-adic setting have been made, e.g. in Mahler [8, 9], Ruban [10], and Schneider [11]. While the two continued fraction algorithms of Ruban and Schneider mimic the classical one more closely than those of Mahler, the two ( $p$-adic) continued fractions of Mahler, which are intentionally constructed via best approximants, naturally yield better approximation results. However, a serious drawback of Mahler's continued fractions in comparison to those of Ruban and Schneider is the lack of representation uniqueness, and this in a way is reflected by the fact that the continued fractions of Ruban and Schneider can be used to characterize rational numbers (see Bundschuh [3] and Laohakosol [6]), but those of Mahler cannot. As seen from [3 and 6], there do exist rational numbers with periodic $p$-adic continued fractions and this indicates difficulties, or probably failures, in obtaining any analogue of Lagrange's classical contribution relating quadratic surds to periodic continued fractions. All in all, we may safely say that no existing $p$-adic continued fraction algorithm possesses as many desirable properties as the classical algorithm.

All is not lost, however, since many other properties such as metric properties do have $p$-adic analogues (see e.g. Ruban [10]). This paper deals with the algebraic independence of Liouville type continued fractions, another aspect in which both the classical and the $p$-adic theories bear similar fruits. In short, we shall obtain certain $p$-adic analogues of results that recently appeared in Adams [1].

\section{Notation.}

$$
\begin{aligned}
p & \text { a fixed rational prime, } \\
||_{p} & \text { the corresponding } p \text {-adic valuation, } \\
& \text { normalized so that }|p|_{p}=1 / p, \\
|| & \text { the usual absolute valuation, } \\
\mathbf{Z} & \text { the ring of rational integers, } \\
\mathbf{Q} & \text { the field of rational numbers, } \\
\mathbf{Z}_{p} & \text { the ring of } p \text {-adic integers, } \\
p \mathbf{Z}_{p} \quad:=\left\{p z ; z \in \mathbf{Z}_{p}\right\} & \\
I & :=\left\{b \in \mathbf{Q} ; b=c_{-j} p^{-j}+c_{-j+1} p^{-j+1}+\cdots+c_{0}\right. \\
& \quad \text { for some positive integer } j, \\
& \left.c_{i} \in\{0,1, \ldots, p-1\} \text { for all } i \text { with } c_{-j} \neq 0\right\} .
\end{aligned}
$$

Received by the editors April 15, 1986 and, in revised form, August 13, 1986.

1980 Mathematics Subject Classification (1985 Revision). Primary 11J61, 11J85, 11 J70.

Key words and phrases. p-adic continued fractions, algebraic independence, Liouville numbers. Partially supported by the 1985-1986 Kasetsart University Research Grant. 
3. Ruban continued fractions (RCF). Following Ruban [10], see also $\S 2$ of Laohakosol [6], any number $\alpha \in p \mathbf{Z}_{p}-\{0\}$ can be uniquely represented by an RCF

$$
\alpha=\frac{1}{b_{0}+\frac{1}{b_{1}+\frac{1}{b_{2}+\cdots}}}:=\operatorname{RCF}\left[b_{0}, b_{1}, b_{2}, \ldots\right],
$$

where each $b_{k}=\sum_{i=-n}^{0} c_{i} p^{i} \in I(n \in \mathbf{N})$ if $1 / \alpha_{k}$ is written as $\sum_{i=-n}^{\infty} c_{i} p^{i}$, and $\alpha_{k+1}=1 / \alpha_{k}-b_{k}, \alpha_{0}=\alpha$, with $c_{i} \in\{0,1, \ldots, p-1\}$. Define

$$
\begin{gathered}
A_{-1}=1, \quad A_{0}=0, \quad B_{-1}=0, \quad B_{0}=1, \\
A_{k+1}=b_{k} A_{k}+A_{k-1}, \quad B_{k+1}=b_{k} B_{k}+B_{k-1} \quad(k=0,1, \ldots) .
\end{gathered}
$$

Then the $k$ th convergent of the RCF of $\alpha$ is

$$
A_{k} / B_{k}=\operatorname{RCF}\left[b_{0}, b_{1}, \ldots, b_{k-1}\right] \quad(k=1,2, \ldots) .
$$

Since the partial quotients, $b_{i}$ 's, $A_{k}$, and $B_{k}$ are all rational, not necessarily integral, it is convenient to introduce an associated continued fraction

$$
\frac{\gamma_{0}}{\beta_{0}+\frac{\gamma_{1}}{\beta_{1}+\frac{\gamma_{2}}{\beta_{2}+\cdots}}}:=\operatorname{RCF}\left[\gamma_{0} / \beta_{0}, \gamma_{1} / \beta_{1}, \gamma_{2} / \beta_{2}, \ldots\right],
$$

where $\gamma_{0}=\left|b_{0}\right|_{p}, \gamma_{n}=\left|b_{n} b_{n-1}\right|_{p}(n \geq 1), \beta_{n}=b_{n}\left|b_{n}\right|_{p}(n \geq 0)$. Clearly, the partial numerators $\gamma_{n}$ and the partial denominators $\beta_{n}$ of the associated continued fraction (3.2) are positive integers. We similarly define the $k$ th convergent of (3.2) to be

$$
A_{k} / B_{k}:=\operatorname{RCF}\left[\gamma_{0} / \beta_{0}, \gamma_{1} / \beta_{1}, \ldots, \gamma_{k-1} / \beta_{k-1}\right] \quad(k=1,2, \ldots),
$$

where $A_{-1}=1, A_{0}=0, B_{-1}=0, B_{0}=1$,

$$
A_{k+1}=\beta_{k} A_{k}+\gamma_{k} A_{k-1}, \quad B_{k+1}=\beta_{k} B_{k}+\gamma_{k} B_{k-1} \quad(k=1,2, \ldots) .
$$

The RCF (3.1) and its associated continued fraction (3.2) are equivalent in the sense that $A_{k} / B_{k}=A_{k} / B_{k}$ for every $k \geq 1$. The following lemma summarizes basic properties of RCF and its associated continued fraction which will be needed later.

LEMMA 1. Let the notation be as set up at the beginning of this section. Then for $k \geq 1$, we have

(i) $\left|\alpha-A_{k} / B_{k}\right|_{p}=\left|\alpha-A_{k} / B_{k}\right|_{p}=\left|b_{0}^{2} b_{1}^{2} \cdots b_{k-1}^{2} b_{k}\right|_{p}^{-1}>0$,

(ii) $M_{k}:=\max \left(A_{k}, B_{k}\right)<p 2^{k-1}\left|b_{0}^{2} b_{1}^{2} \cdots b_{k-2}^{2} b_{k-1}\right|_{p}$.

PROOF. For the proof of (i), see e.g. Laohakosol [6]. To prove (ii), from definition, we see that

$$
1 \leq \beta_{k} \leq \gamma_{k}-1 \quad(k \geq 1)
$$

This together with the fact that the sequences of positive integers $\left(A_{k}\right),\left(B_{k}\right)$ and hence $\left(M_{k}\right)$ are strictly increasing imply, using also (3.3),

$$
M_{k+1} \leq\left(2 \gamma_{k}-1\right) M_{k} \quad(k \geq 1) .
$$

By induction, using this last inequality and the recurrence relations (3.3), (ii) immediately follows. 
4. Schneider continued fractions (SCF). Following Schneider [11], see also Bundschuh [3], any number $\alpha \in p \mathbf{Z}_{p}-\{0\}$ can be uniquely represented by an SCF

$$
\alpha=\frac{a_{0}}{b_{0}+\frac{a_{1}}{b_{1}+\frac{a_{2}}{b_{2}+\cdots}}}:=\operatorname{SCF}\left[a_{0} / b_{0}, a_{1} / b_{1}, a_{2} / b_{2}, \ldots\right],
$$

where each $a_{i}=p^{s}$ for some positive integer $s=s(i)$ and $b_{i} \in\{1,2, \ldots, p-1\}$ if $\alpha_{k}$ is written as $\sum_{i=-s}^{\infty} c_{i} p^{i}$, and

$$
\alpha_{k}=\frac{a_{k}}{b_{k}+\alpha_{k+1}}, \quad \alpha_{0}=\alpha
$$

Define

$$
\begin{aligned}
A_{-1}=1, \quad B_{-1} & =0, \quad A_{0}=0, \quad B_{0}=1 \\
A_{k+1}=b_{k} A_{k}+a_{k} A_{k-1}, & B_{k}=b_{k} B_{k}+a_{k} B_{k-1} \quad(k=0,1,2, \ldots) .
\end{aligned}
$$

Then

$$
A_{k} / B_{k}=\operatorname{SCF}\left[a_{0} / b_{0}, \ldots, a_{k-1} / b_{k-1}\right] \quad(k=1,2, \ldots)
$$

represents the $k$ th convergent of the SCF of $\alpha$. The next lemma summarizes basic properties of SCF to be used later.

LEMMA 2. Let the notation be as set up the beginning of this section. Then for $k \geq 1$, we have

(i) $\left|\alpha-A_{k} / B_{k}\right|_{p}=\left(a_{0} a_{1} \cdots a_{k}\right)^{-1}>0$,

(ii) $M_{k}:=\max \left(A_{k}, B_{k}\right)<a_{0} a_{1} \cdots a_{k-1}$.

PrOOF. By induction, we have

$$
\alpha-A_{k} / B_{k}=\frac{(-1)^{k} a_{0} a_{1} \cdots a_{k}}{B_{k}\left(\alpha_{k} B_{k}+a_{k} B_{k-1}\right)} \quad(k \geq 0),
$$

where $\alpha_{k}=b_{k}+\operatorname{SCF}\left[a_{k+1} / b_{k+1}, a_{k+2} / b_{k+2}, \ldots\right]$. (i) now follows by noting that for each $k$,

$$
\left|B_{k}\right|_{p}=\left|\alpha_{k}\right|_{p}=1, \quad\left|a_{k}\right|_{p}<1 .
$$

(ii) is easily checked by induction.

5. Liouville type algebraic independence. In this section, we establish a $p$-adic analogue of a recent Liouville type algebraic independence criterion of Adams [1]. The proof is quite close to that given in Adams [1] (see also Flicker [5], Laohakosol and Ubolsri [7], Shiokawa [12]).

THEOREM 3. Let $\alpha_{1}, \alpha_{2}, \ldots, \alpha_{n}$ be $n$ numbers in $p \mathbf{Z}_{p}-\{0\}$. Let $A_{N, j}, B_{N, j}$ $(N=1,2, \ldots ; j=1,2, \ldots, n)$ be rational integers with

$$
M_{N, j}=\max \left(\left|A_{N, j}\right|,\left|B_{N, j}\right|\right) \rightarrow \infty \quad(N \rightarrow \infty) .
$$

For $j=2, \ldots, n$, assume that

$$
\lim _{N \rightarrow \infty} \frac{\left|\alpha_{j-1}-A_{N, j-1} / B_{N, j-1}\right|_{p}}{\left|\alpha_{j}-A_{N, j} / B_{N, j}\right|_{p}}=0 .
$$


Further assume that for each $j=1,2, \ldots, n$ and all positive integers $D$, there is an $N_{0}=N_{0}(D)$ such that for all $N>N_{0}$,

$$
0<\left|\alpha_{j}-A_{N, j} / B_{N, j}\right|_{p}<\left(M_{N, 1} \cdots M_{N, j}\right)^{-D} .
$$

Then $\alpha_{1}, \alpha_{2}, \ldots, \alpha_{n}$ are algebraically independent.

PROOF. We proceed by induction on $n$. For $n=1,(5.2)$ implies that $\alpha_{1}$ is a $p$-adic Liouville number (see Mahler $[9$, p. 56]). It is thus transcendental, and we are done in this case. Now consider $n>1$. Assume that the result were false; then there would exist a nonzero polynomial $P\left(X_{1}, \ldots, X_{n}\right)$ with rational integral coefficients of minimal total degree such that $P\left(\alpha_{1}, \alpha_{2}, \ldots, \alpha_{n}\right)=0$. Expanding the polynomial $P$ about $\alpha_{1}, \ldots, \alpha_{n}$, we get

$$
P\left(X_{1}, \ldots, X_{n}\right)=\sum c_{(\nu)}\left(X_{1}-\alpha_{1}\right)^{\nu_{1}} \cdots\left(X_{n}-\alpha_{n}\right)^{\nu_{n}}
$$

where $(\nu)=\left(\nu_{1}, \ldots, \nu_{n}\right)$. For $i=1, \ldots, n$, set $C_{i}=c_{(\nu)}$ where $(\nu)$ has a 1 in the $i$ th coordinate and a 0 everywhere else. We thus have

$$
C_{n}=\frac{\partial}{\partial X_{n}} P\left(\alpha_{1}, \ldots, \alpha_{n}\right) \text {. }
$$

We claim that $C_{n} \neq 0$. For $C_{n}=0$ would imply $\partial P\left(X_{1}, \ldots, X_{n}\right) / \partial X_{n} \equiv 0$, or else there would exist a nonzero polynomial of smaller total degree than $P$ vanishing at $\alpha_{1}, \ldots, \alpha_{n}$. But then $X_{n}$ did not occur in the polynomial $P$ and we obtain that $\alpha_{1}, \ldots, \alpha_{n-1}$ are algebraically dependent, contradicting the induction hypothesis, and the claim is verified. Now putting

$$
\delta_{i}(N)=A_{N, i} / B_{N, i}-\alpha_{i} \quad(i=1, \ldots, n),
$$

we get

$$
\begin{aligned}
& P\left(A_{N, 1} / B_{N, 1}, \ldots, A_{N, n} / B_{N, n}\right) \\
& =\delta_{n}(N)\left\{\left(C_{1} \delta_{1}(N) / \delta_{n}(N)+\cdots\right.\right. \\
& \left.\left.\quad+C_{n-1} \delta_{n-1}(N) / \delta_{n}(N)+C_{n}\right)+O\left(\left|\delta_{n}(N)\right|_{p}\right)\right\}
\end{aligned}
$$

where $\left|\delta_{n}(N)\right|_{p}=\max \left(\left|\delta_{i}(N)\right|_{p} ; i=1, \ldots, n\right)$ by (5.1). The expression inside the curly bracket is not 0 for $N$ large because of (5.1) and the fact that $C_{n} \neq 0$. Since $\delta_{n}(N) \neq 0$, we thus see that

$$
P\left(A_{N, 1} / B_{N, 1}, \ldots, A_{N, n} / B_{N, n}\right) \neq 0 \text { for sufficiently large } N \text {. }
$$

Now denote by $D_{1}, \ldots, D_{n}$ the degree of $P$ in $X_{1}, \ldots, X_{n}$, respectively. Thus

$$
B_{N, 1}^{D_{1}} B_{N, 2}^{D_{2}} \cdots B_{N, n}^{D_{n}} P\left(A_{N, 1} / B_{N, 1}, \ldots, A_{N, n} / B_{N, n}\right)
$$

is a nonzero rational integer and so

$$
\left|B_{N, 1}^{D_{1}} \cdots B_{N, n}^{D_{n}} P\left(A_{N, 1} / B_{N, 1}, \ldots, A_{N, n} / B_{N, n}\right)\right| \leq K M_{N, 1}^{D_{1}} \cdots M_{N, n}^{D_{n}},
$$

where $K=K\left(\alpha_{1}, \ldots, \alpha_{n}, P\right)$ is a constant. By the product formula, we then have

$$
\begin{aligned}
& \left|P\left(A_{N, 1} / B_{N, 1}, \ldots, A_{N, n} / B_{N, n}\right)\right|_{p} \\
& \quad \geq\left|B_{N, 1}^{D_{1}} \cdots B_{N, n}^{D_{n}} P\left(A_{n, 1} / B_{N, 1}, \ldots, A_{N, n} / B_{N, n}\right)\right|_{p} \\
& \quad \geq\left(K M_{N, 1}^{D_{1}} \cdots M_{N, n}^{D_{n}}\right)^{-1} .
\end{aligned}
$$


On the other hand, by the strong triangle inequality and (5.3), we get for all $N$ large,

$$
\begin{aligned}
\left|P\left(A_{N, 1} / B_{N, 1}, \ldots, A_{N, n} / B_{N, n}\right)\right|_{p} & \leq\left|C_{n} \delta_{n}(N)\right|_{p} \\
& \leq K^{\prime}\left|\alpha_{n}-A_{N, n} / B_{N, n}\right|_{p}
\end{aligned}
$$

where $K^{\prime}=K^{\prime}\left(\alpha_{1}, \ldots, \alpha_{n}, P\right)$ is a constant. Now (5.4) and (5.5) together violate (5.2) and this completes the proof.

REMARKS. There is another similar algebraic independence criterion due to Bundschuh and Wallisser [4, Corollary 2]. It may be of interest to state and compare it with our result above. For simplicity, we only consider the case of two numbers.

THEOREM (BUNDSCHUH AND WALLISSER). Let $\alpha_{1}, \alpha_{2}$ be two numbers in $p \mathbf{Z}_{p}-\{0\}$. To each natural number $D$, if there exist rational integers $A_{1}, B_{1}$ $(\neq 0), A_{1}, B_{2}(\neq 0)$ such that

$$
\begin{gathered}
\left|\alpha_{1} B_{1}-A_{1}\right|_{p}<(2 M)^{-2 D} \\
0<\left|\alpha_{2} B_{2}-A_{2}\right|_{p}<(2 M)^{-2 D}\left|\alpha_{1} B_{1}-A_{1}\right|_{p}^{D},
\end{gathered}
$$

where $M=\max \left(\left|A_{1}\right|,\left|B_{1}\right|,\left|A_{2}\right|,\left|B_{2}\right|\right)$, then $\alpha_{1}$ and $\alpha_{2}$ are algebraically independent.

(Originally, Bundschuh and Wallisser proved this result with $B_{1}=B_{2}$.)

Though Bundschuh and Wallisser's criterion requires only two pairs of approximants $A_{1} / B_{1}$ and $A_{2} / B_{2}$, rather than two sequences as in Theorem 3 , the two conditions (5.1) and (5.2) are evidently more relaxed than (5.6) and (5.7). Consequently, we shall briefly note at the ends of the next two sections that the classes of algebraically independent ( $p$-adic) continued fractions obtainable via Theorem 3 are larger than those obtainable through Bundschuh and Wallisser's criterion.

6. Liouville type RCFs. In this section, we apply Theorem 3 to construct a class of algebraically independent RCFs of Liouville type.

THEOREM 4. Let $\alpha_{1}, \ldots, \alpha_{n}$ be $n$ numbers in $p \mathbf{Z}_{p}-\{0\}$ with RCFs

$$
\alpha_{j}=\mathrm{RCF}\left[b_{0, j}, b_{1, j}, b_{2, j}, \ldots\right] \quad(j=1, \ldots, n) .
$$

Suppose there are constants $\tau, r>1$ and a function $g(i)$ for $i=1,2, \ldots$ with $g(i) \rightarrow \infty(i \rightarrow \infty)$, and a subsequence of positive integers $N_{1}<N_{2}<\cdots$ such that for all $N=0,1, \ldots$ and $j=2,3, \ldots, n$ we have

$$
\begin{gathered}
\left|b_{N, 1}\right|_{p} \geq\left(\sqrt{2}\left|b_{N-k, 1}\right|_{p}\right)^{\tau^{k}} \quad(k=1, \ldots, N) \\
\left|b_{N, j-1}\right|_{p} \geq r\left|b_{N, j}\right|_{p} \\
\left|b_{N_{i}, j}\right|_{p} \geq\left|b_{N_{i}-1,1}\right|_{p}^{g(i)}
\end{gathered}
$$

Then $\alpha_{1}, \ldots, \alpha_{n}$ are algebraically independent.

ProOF. We need only verify the hypotheses of Theorem 3 with the $N$ of Theorem 3 replaced by the $N_{i}$ above. To work with rational integers, rather than rationals, we have to resort to associated continued fractions as described in $\S 3$. Let $A_{k, j} / B_{k, j}$ and $A_{k, j} / B_{k, j}(k=1,2, \ldots ; j=1, \ldots n)$ be the $k$ th convergents of 
the RCF of $\alpha_{j}$ and of its associated continued fraction, respectively. To verify (5.1), we note from Lemma 1(i), the equivalence of associated RCF, and (6.2) that

$$
\begin{aligned}
\frac{\left|\alpha_{j-1}-A_{N_{i}, j-1} / B_{N_{i}, j-1}\right|_{p}}{\left|\alpha_{j}-A_{N_{i}, j} / B_{N_{i}, j}\right|_{p}} & =\frac{\left|\alpha_{j-1}-A_{N_{i}, j-1} / B_{N_{i}, j-1}\right|_{p}}{\left|\alpha_{j}-A_{N_{i}, j} / B_{N_{i}, j}\right|_{p}} \\
& =\frac{\left|b_{0, j}^{2} b_{1, j}^{2} \cdots b_{N_{i}-1, j}^{2} b_{N_{i}, j}\right|_{p}}{\left|b_{0, j-1}^{2} b_{1, j-1}^{2} \cdots b_{N_{i}-1, j-1}^{2} b_{N_{i}, j-1}\right|_{p}} \\
& \leq 1 / r^{2 N_{i}+1} \rightarrow 0 \quad(i \rightarrow \infty) .
\end{aligned}
$$

To verify (5.2), we see from Lemma 1 (i) that

$$
0<\left|\alpha_{j}-A_{N, j} / B_{N, j}\right|_{p}=\left|b_{0, j}^{2} b_{1, j}^{2} \cdots b_{N-1, j}^{2} b_{N, j}\right|_{p}^{-1},
$$

and note that it suffices to show that for sufficiently large $i$

$$
\left|b_{0, j}^{2} b_{1, j}^{2} \cdots b_{N_{i}-1, j}^{2} b_{N_{i}, j}\right|_{p} \geq\left(M_{N_{i}, 1} M_{N_{i}, 2} \cdots M_{N_{i}, j}\right)^{D},
$$

where $M_{k, j}=\max \left(A_{k, j}, B_{k, j}\right)>0$, and $D$ is a large but fixed positive integer. By Lemma 1 (ii) and (6.2), for $t=1, \ldots, j$, we have

$$
\begin{aligned}
M_{N_{i}, t} & <p 2^{N_{i}-1}\left|b_{0, t}^{2} b_{1, t}^{2} \cdots b_{N_{i}-2, t}^{2} b_{N_{i}-1, t}\right|_{p} \quad(t=1, \ldots, j) \\
& \leq p 2^{N_{i}-1} \frac{1}{r^{(t-1)\left(2 N_{i}-1\right)}}\left|b_{0,1}^{2} b_{1,1}^{2} \cdots b_{N_{i}-2,1}^{2} b_{N_{i}-1,1}\right|_{p} \\
& \leq p 2^{N_{i}-1}\left|b_{0,1}^{2} b_{1,1}^{2} \cdots b_{N_{i}-2,1}^{2} b_{N_{i}-1,1}\right|_{p} .
\end{aligned}
$$

Thus

$$
\begin{aligned}
\left(M_{N_{i}, 1} \cdots M_{N_{i}, j}\right)^{D} & <\left(p^{j} 2^{j\left(N_{i}-1\right)}\left|b_{0,1}^{2} b_{1,1}^{2} \cdots b_{N_{i}-2,1}^{2} b_{N_{i}-1,1}\right|_{p}^{j}\right)^{D} \\
& \leq p^{j D}\left|b_{N_{i}-1,1}^{1+2 / \tau+\cdots+2 / \tau^{N_{i}-1}}\right|_{p}^{j D} \\
& \leq p^{j D}\left|b_{N_{i}-1,1}\right|_{p}^{2 j \tau D /(\tau-1)} .
\end{aligned}
$$

But by (6.3), this last term is majorized by the left-hand expression of (6.4) when $i$ is sufficiently large, and we are done.

REMARKS. Referring to the remarks at the end of $\S 5$, in contrast to (6.1), (6.2), and (6.3), Bundschuh and Wallisser's criterion would require something like

$$
\left|b_{N_{i}, 1}\right|_{p} \geq\left(p\left|b_{N_{i}-1,2}\right|_{p}\right)^{f(i)}, \quad\left|b_{N_{i}, 2}\right|_{p} \geq\left|b_{N_{i}, 1}\right|_{p}^{g(i)}
$$

where $f(i)$ and $g(i) \rightarrow \infty(i \rightarrow \infty)$. Clearly, our Theorem 4 yields a bigger class of algebraically independent RCFs.

7. Liouville type SCFs. In this section, we exhibit a class of algebraically independent SCFs of Liouville type. Since the idea of the proof is the same as that of Theorem 4, we give the full statement of the theorem, but merely sketch its proof.

THEOREM 5. Let $\alpha_{1}, \ldots, \alpha_{n}$ be $n$ numbers in $p \mathbf{Z}_{p}-\{0\}$ with SCFs

$$
\alpha_{j}=\operatorname{SCF}\left[a_{0, j} / b_{0, j}, a_{1, j} / b_{1, j}, a_{2, j} / b_{2, j}, \ldots\right] \quad(j=1, \ldots, n) .
$$

Suppose there are constants $\tau, r>1$ and a function $g(i)$ for $i=1,2, \ldots$ with $g(i) \rightarrow \infty(i \rightarrow \infty)$ and a subsequence of positive integers $N_{1}<N_{2}<\cdots$ such 
that, for all $N=0,1, \ldots$ and $j=2,3, \ldots, n$, we have

$$
\begin{aligned}
a_{N, 1} & \geq a_{N-k, 1}^{\tau^{k}} \quad(k=1, \ldots, N), \\
a_{N, j-1} & \geq r a_{N, j}, \\
a_{N_{i}, j} & \geq a_{N_{i}-1,1}^{g(i)} .
\end{aligned}
$$

Then $\alpha_{1}, \ldots, \alpha_{n}$ are algebraically independent.

SKETCH OF THE PROOF. To verify (5.1), we note that

$$
\begin{aligned}
\frac{\left|\alpha_{j-1}-A_{N_{i}, j-1} / B_{N_{i}, j-1}\right|_{p}}{\left|\alpha_{j}-A_{N_{i}, j} / B_{N_{i}, j}\right|_{p}} & =\frac{a_{0, j} a_{1, j} \cdots a_{N_{i}, j}}{a_{0, j-1} a_{1, j-1} \cdots a_{N_{i}, j-1}} \\
& \leq r^{-\left(N_{i}-1\right)} \rightarrow 0 \quad(i \rightarrow \infty) .
\end{aligned}
$$

From

$$
0<\left|\alpha_{j}-A_{N, j} / B_{N, j}\right|_{p}=\left(a_{0, j} a_{1, j} \cdots a_{N, j}\right)^{-1},
$$

to verify (5.2), it suffices to show for $i$ sufficiently large

$$
a_{0, j} a_{1, j} \cdots a_{N_{i}, j} \geq\left(M_{N_{i}, 1} M_{N_{i}, 2} \cdots M_{N_{i}, j}\right)^{D} .
$$

This follows from the following sequence of inequalities.

$$
\begin{aligned}
M_{N_{i}, t} & <a_{0, t} a_{1, t} \cdots a_{N_{i}-1, t} \quad(t=1, \ldots, j) \\
& \leq a_{0,1} a_{1,1} \cdots a_{N_{i}-1,1} r^{-(t-1) N_{i}} \\
& \leq a_{0,1} a_{1,1} \cdots a_{N_{i}-1,1} \\
\left(M_{N_{i}, 1} \cdots M_{N_{i}, j}\right)^{D} & <\left(a_{0,1} a_{1,1} \cdots a_{N_{i}-1,1}\right)^{j D} \\
& \leq\left(a_{N_{i}-1,1}^{1+1 / \tau+1 / \tau^{N_{i}-1}}\right)^{j D}<a_{N_{i}-1,1}^{j \tau D /(\tau-1)}
\end{aligned}
$$

REMARKS. As with the remarks at the end of $\S 6$, in contrast to (7.1), (7.2), and (7.3), Bundschuh and Wallisser's criterion would require something like

$$
a_{N_{i}, 1} \geq a_{N_{i}-1,2}^{f(i)}, \quad a_{N_{i}, 2} \geq a_{N_{i}, 1}^{g(i)}
$$

and clearly, our Theorem 5 gives a bigger class of algebraically independent SCFs. Some discussion and examples of continued fractions constructed via Bundschuh and Wallisser's criterion can also be found in Bundschuh [2].

ACKNOWLEDGMENT. We wish to thank the referee for pointing out some errors and suggesting an improvement in the earlier presentation of the paper.

\section{REFERENCES}

1. W. W. Adams, The algebraic independence of certain Liouville continued fractions, Proc. Amer. Math. Soc. 95 (1985), 512-516.

2. P. Bundschuh, Fractions continues et independance algébrique en p-adique, Asterisque 41-42 (1977), 179-181.

3. __ p-adische Kettenbrüche und Irrationalität p-adischer Zahlen, Elem. Math. 32 (1977), $36-40$.

4. P. Bundschuh und R. Wallisser, Algebraische Unabhängigkeit p-adischer Zahlen, Math. Ann. 221 (1976), 243-249.

5. Y. Flicker, Algebraic independence by a method of Mahler, J. Austral. Math. Soc. Ser. A 27 (1979), 173-188. 
6. V. Laohakosol, A characterization of rational numbers by $p$-adic Ruban continued fractions, J. Austral. Math. Soc. Ser. A 39 (1985), 300-305.

7. V. Laohakosol and P. Ubolsri, Some algebraically independent continued fractions, Proc. Amer. Math. Soc. 95 (1985), 169-173.

8. K. Mahler, Zur Approximation p-adischer Irrationalzahlen, Nieuw Arch. Wisk. 18 (1934), 2234.

9. ___ Lectures on diophantine approximations. Part 1, g-adic numbers and Roth's Theorem, Univ. of Notre Dame Press, 1961.

10. A. Ruban, Certain metric properties of the p-adic numbers, Sibirsk. Mat. Zh. 11 (1970), 222227; English transl., Siberian Math. J. 11 (1970), 176-180.

11. Th. Schneider, Über p-adische Kettenbrüche, Symposia Math. 4 (1970), 181-189.

12. I. Shiokawa, Algebraic independence of certain gap series, Arch. Math. 38 (1982), 438-442.

Department of Mathematics, Kasetsart University, Bangkok 10900 , THAILAND 